\title{
Modeling of light propagation and molecules alignment in nematic liquid crystals of planar orientation
}

\author{
Filip A. Sala*, Mirosław A. Karpierz \\ Faculty of Physics, Warsaw University of Technology, Koszykowa 75, 00-662 Warszawa
}

Received February 10, 2012; accepted March 23, 2012; published March 31, 2012

\begin{abstract}
In this work the results of light propagation in nematic liquid crystals of planar orientation are presented. For calculations a fully vectorial Beam Propagation Method is used combined with a model describing molecules orientation We focus on a comparison between one- and two-angle model describing director orientation. Differences are also shown in light propagation and polarization of the propagated beams.
\end{abstract}

Nematic liquid crystals are unique materials with vast nonlinearities, caused by molecules reorientation. This property gives a possibility of forming non-diffractive beams called nematicons. Such beams were observed in experiments many times and predicted theoretically [1-4]. Liquid crystals are anisotropic materials and thus to perform simulations a fully vectorial Beam Propagation Method has to be used [5-6]. As the optical field interacts with molecules and forces them to reorient also director orientation has to be calculated during the propagation. In most simulations to date, orientation of the molecules were described only by one angle: twist for planar configurations or azimuthal for homeotropic ones. In this paper we use two angles to describe director orientation and compare the results of light propagation between one- and two-angle models. Due to our studies only in particular cases oneangle approximation can be assumed.

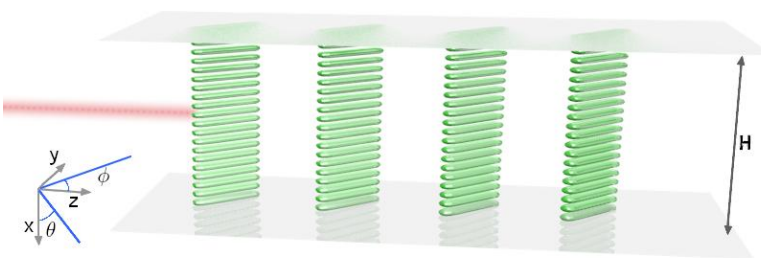

Fig. 1. The analyzed setup and the coordinate system.

We analyze a cell of height $\mathrm{H}=50 \mu \mathrm{m}$, filled with liquid crystals which parameters correspond to the low birefringent liquid crystals (1550) synthesized by Prof. Dąbrowski's laboratory at the Military University of Technology in Warsaw. Molecules configuration is planar with anchoring at some angle $0 \leq \varphi_{0} \leq 90^{\circ}$ to the $\mathrm{z}$-axis in yz plane. The beam of a Gaussian shape of FWHM=5.5 $\mu \mathrm{m}$ and wavelength $\lambda=532 \mathrm{~nm}$ is launched into the system. Light is propagated along the $\mathrm{z}$-axis (Fig. 1).

In simulations the Full-Vector Beam Propagation Method (FV-BPM) is used with no paraxial approximation. The model describing director orientation is based on the Frank-Oseen equation on free energy density [7]:

$$
\begin{aligned}
& f=\frac{1}{2} K_{11}(\nabla \vec{n})^{2}+\frac{1}{2} K_{22}(\vec{n} \cdot(\nabla \times \vec{n}))^{2}+ \\
& +\frac{1}{2} K_{33}(\vec{n} \times(\nabla \times \vec{n}))^{2}-\frac{1}{2} \Delta \varepsilon \varepsilon_{0}(\vec{n} \cdot \vec{E})^{2}
\end{aligned}
$$

where $K_{\mathrm{ii}}$ are (Frank) elastic constants corresponding to the splay, twist and bend deformations, director $\vec{n}$ is defined as: $\vec{n}=\left[\begin{array}{lll}\cos \theta ; & \sin \theta \sin \varphi ; & \sin \theta \cos \varphi\end{array}\right], \varphi-$ twist angle (in yz plane), $\theta$-azimuthal angle, $\Delta \varepsilon$ is an optical anisotropy.

We use a single constant approximation $K_{11}=K_{22}=K_{33}=K$ so Eq. (1) can be simplified to the form:

$$
f=\frac{1}{2} K \cdot\left[(\nabla \vec{n})^{2}+(\nabla \times \vec{n})^{2}\right]-\frac{1}{2} \Delta \varepsilon \varepsilon_{0}(\vec{n} \cdot \vec{E})^{2} .
$$

Moreover, derivatives with respect to $z$ are neglected which means that paraxial approximation is used. Equation (2) is substituted into Euler-Lagrange equations form which we obtain two equations describing director orientation in a transverse plane:

$$
\begin{aligned}
\sin ^{2} \theta \cdot( & \left.\frac{\partial^{2} \varphi}{\partial x^{2}}+\frac{\partial^{2} \varphi}{\partial y^{2}}\right)+\sin 2 \theta\left(\frac{\partial \varphi}{\partial x} \frac{\partial \theta}{\partial x}+\frac{\partial \varphi}{\partial y} \frac{\partial \theta}{\partial y}\right)+ \\
& \frac{\Delta \varepsilon \varepsilon_{0}}{2 K}\left(2 E_{y} E_{z} \sin ^{2} \theta \cos 2 \varphi+\right. \\
+ & \sin 2 \theta\left(E_{x} E_{y} \cos \varphi-E_{x} E_{y} \sin \varphi\right)+ \\
+ & \left.\sin ^{2} \theta \sin 2 \varphi\left(\left|E_{y}\right|^{2}-\left|E_{z}\right|^{2}\right)\right)=0
\end{aligned}
$$

$$
\begin{gathered}
\left(\frac{\partial^{2} \theta}{\partial x^{2}}+\frac{\partial^{2} \theta}{\partial y^{2}}\right)-\frac{1}{2} \sin 2 \theta\left(\left(\frac{\partial \varphi}{\partial x}\right)^{2}+\left(\frac{\partial \varphi}{\partial y}\right)^{2}\right)+ \\
+\frac{\Delta \varepsilon \varepsilon_{0}}{2 K}\left(E_{z} E_{y} \sin 2 \theta \sin 2 \varphi+\right. \\
+2 \cos 2 \theta\left(E_{x} E_{y} \sin \varphi+E_{x} E_{z} \cos \varphi\right)+ \\
\left.+\sin 2 \theta\left(\left|E_{z}\right|^{2} \cos ^{2} \varphi+\left|E_{y}\right|^{2} \sin ^{2} \varphi-\left|E_{x}\right|^{2}\right)\right)=0 .
\end{gathered}
$$

*E-mail: sala@if.pw.edu.pl 
The electric field components are complex. In our notation $E_{\mathrm{i}} E_{\mathrm{j}}=E_{\mathrm{i}}^{*} E_{\mathrm{j}}+E_{\mathrm{i}} E_{\mathrm{j}}^{*}$ where $\mathrm{i}, \mathrm{j} \in\{x, y, z\}$. These equations are solved with relaxation method. Calculations are carried out on a 2D transverse grid of $200 \times 200$ points. Molecular reorientation is calculated in separate transverse "slices" as there is no derivative with respect to $z$. The light field is calculated step by step along the $\mathrm{z}$-axis. The integration steps are set as follows: $\Delta z=10 \mathrm{~nm}$ and $\Delta x=\Delta y=250 \mathrm{~nm}$. Elastic constant $K=12 \mathrm{pN}$ is assumed. The anisotropy of liquid crystals is $\Delta n=0.06$.

At first, we will analyze only director reorientation with no beam propagation. The electric field of a shape corresponding to the Gaussian beam of the following for is used:

$$
\begin{aligned}
& E_{x}=A \exp \left(-\frac{x^{2}+y^{2}}{2 \sigma_{0}^{2}}\right) \sin \alpha \\
& H_{y}=\frac{\sqrt{\varepsilon_{r}}}{Z_{0}} E_{x} \\
& E_{y}=A \exp \left(-\frac{x^{2}+y^{2}}{2 \sigma_{0}^{2}}\right) \cos \alpha \\
& H_{x}=-\frac{\sqrt{\varepsilon_{r}}}{Z_{0}} E_{y}
\end{aligned}
$$

where $A$ is amplitude, $\sigma_{0}=\frac{F W H M}{2 \sqrt{2 \ln 2}}$ - beam waist, $Z_{0}=\sqrt{\mu_{0} \varepsilon_{0}^{-1}}$ - impedance of vacuum, $\alpha$-polarization angle.

When the field is y-polarized and $0 \leq \varphi_{0} \leq 90^{\circ} \quad \theta_{0}=90^{\circ}$ (i.e. molecules lie in the yz plane) the molecules reorient in the yz plane and tend to align along the $y$-axis. Such reorientation corresponds only to the twist deformation so one-angle model can be used. When $\varphi_{0}=0$ reorientation will occur only for power above Freedericksz threshold. When the field is $\mathrm{x}$ polarized and $0 \leq \varphi_{0} \leq 90^{\circ}$ reorientation also occurs above the threshold. In such a case the reorientation is only azimuthal and a simple one-angle model can be used. But when it comes to propagation even if the input beam is polarized along $\mathrm{x}$ or $\mathrm{y}$ direction all $\mathrm{x}, \mathrm{y}$ and $\mathrm{z}$ field components appear. Moreover, $\mathrm{x}$-polarized light is diffracted and some part is changed into y-polarized one. Therefore one-angle model is sufficient only in two cases: for the ypolarized light when $0 \leq \varphi_{0} \leq 90^{\circ}, \theta_{0}=90^{\circ}$ and for the $\mathrm{X}$-polarized light.

In other cases, both twist and azimuthal reorientation should be taken into account. Moreover, for instance, for the beam polarized at $45^{\circ}\left(E_{\mathrm{x}}=E_{\mathrm{y}}\right)$ both reorientations are very strong and thus differences between one- and two-angle models are significant (see Fig. 2). In such a case a two-angle model has to be used.
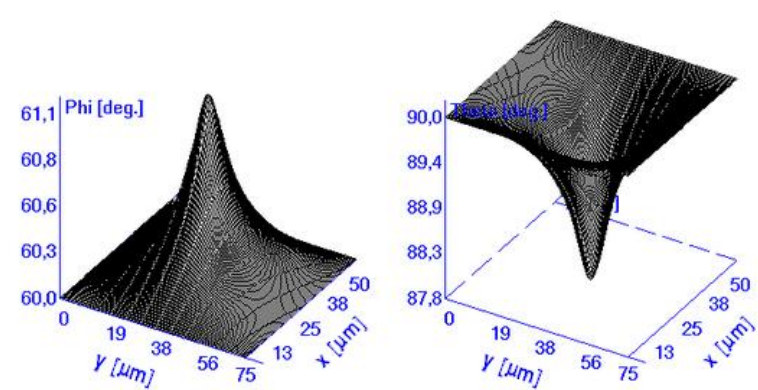

Fig. 2. Director orientation: twist in yz-plane (left) and azimuthal (right) for planar configuration $\varphi_{0}=60^{\circ}$ and input beam polarization $\alpha=45^{\circ}$.

Now we will consider the planar configuration $\varphi_{0}=60^{\circ}$ with a Gaussian beam launched into the system. The beam is described by Eq. (3), and polarized at $\alpha=45^{\circ}$ to the $y$-axis. While increasing power, diffraction is limited and a non-diffractive beam (nematicon) can be formed for $P \approx 20 \mathrm{~mW}$. Using a one-angle model leads to the formation of a soliton which carries much less power, approximately $10 \mathrm{~mW}$ in comparison to a soliton simulated by using a two-angle model which carries approximately $18.5 \mathrm{~mW}$ at the output after $500 \mu \mathrm{m}$ of propagation (see. Fig. 3).

(a)

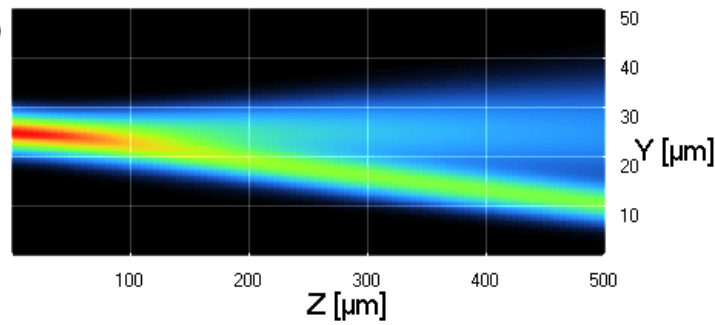

(b)

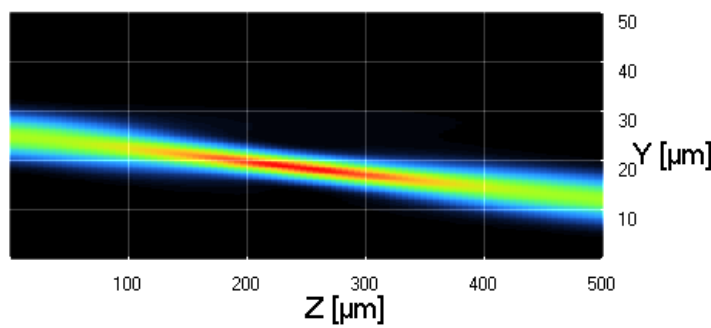

Fig. 3. Light propagation in the yz plane for the light polarized at $45^{\circ}$. and molecules oriented at $\varphi_{0}=60^{\circ}$ to the z-axis. Calculations were made using (a) a one-angle model, (b) a two-angle model. The input beam power is $20 \mathrm{~mW}$ in both cases.

Molecular reorientation for this case is shown in Fig. 4. In a one-angle model the twist reorientation is much weaker than in a two-angle model.

In a one-angle model a large part of energy is diffracted and propagates on-axis while a soliton in both cases is formed off-axis. The walk-off angle in simulations performed using a one-angle model is $\delta \approx 2.17^{\circ}$ and 
while using a two-angle model $\delta \approx 1.43^{\circ}$. Theoretically predicted walk-off in this configuration is $\delta \approx 1.76^{\circ}$.

(a)

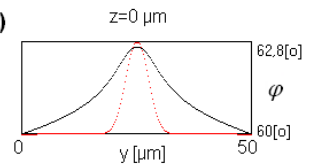

(b)

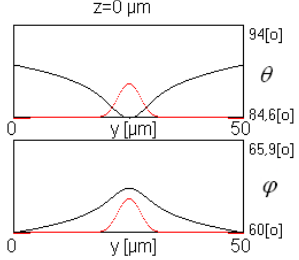

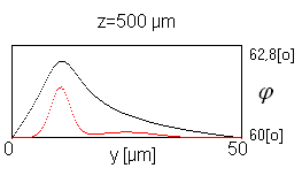

$\mathrm{z}=500 \mu \mathrm{m}$

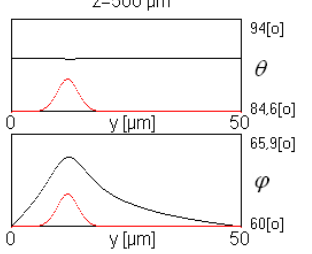

Fig 4. Molecular reorientation plotted along the y-axis for the case from Fig. 3 at the beginning of propagation (left) and at the end $\mathrm{z}=500 \mu \mathrm{m}$ (right): (a) one-angle model (b) two-angle model. The black line is the molecular reorientation and the red line is the normalized optical field intensity.

(a)

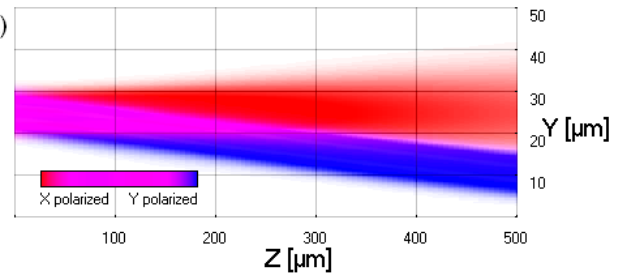

(b)

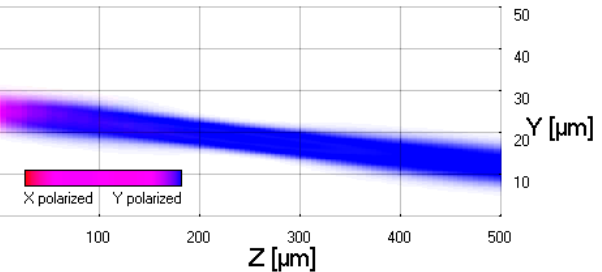

Fig. 5. Polarization distribution in the yz plane. The input beam is polarized at $45^{\circ}$ and molecules are rotated at $\varphi_{0}=60^{\circ}$. The input beam power is $20 \mathrm{~mW}$. The $\mathrm{x}$-polarized light is marked as red and the y-polarized light as blue. Calculations were made using (a) one-angle model (b) two-angle model.
When analyzing polarization of the propagated beams it is visible (Fig. 5) that the X-polarized light goes straight ahead on-axis and the y-polarized light forming a soliton goes off-axis. In a one-angle model the x-polarized part carries much more energy than in a two-angle model.

Concluding, the results of light propagation in liquid crystals of planar configuration have been presented. A comparison has been shown between one- and two-angle models describing director orientation in the context of optical field propagation. Due to our studies, a one-angle model is sufficient in a very few cases. Differences appear in power forming a soliton and proportions between $\mathrm{x}$ - and $\mathrm{y}$-polarized light. Thus a two-angle model should be used when possible, especially when it comes to quantitative simulations.

This work was financially supported by the National Science Centre. Filip Sala was supported by a scholarship from the Center for Advanced Studies.

\section{References}

[1] U.A. Laudyn, M. Kwasny, M.A. Karpierz, Appl. Phys. Lett. 94, 091110 (2009).

[2] U.A. Laudyn, M. Kwaśny, K. Jaworowicz, K.A. Rutkowska, M.A. Karpierz, G. Assanto, Photon. Lett. Poland 1, 7 (2009).

[3] G. Assanto, M.A. Karpierz, Liq. Cryst. 36, 1161 (2009).

[4] G. Assanto, M. Peccianti, C. Conti, Opt. Photon. News 14, 44 (2003).

[5] G.D. Ziogos, E.E. Kriezis, Opt. Quant. Electron 40, 10 (2008).

[6] F.A. Sala, M.A. Karpierz, Photon. Lett. Poland 1, 163 (2009).

[7] I-C Khoo, Liquid crystals (John Wiley \& Sons, Inc 2007). 